Portland State University

PDXScholar

Engineering and Technology Management

Faculty Publications and Presentations

\title{
Identify the Best Alternatives to Help the Diffusion of Teleconsultation by Using the Hierarchical Decision Model (HDM)
}

Hamad A. Alanazi

Portland State University

Tugrul Unsal Daim

Portland State University, tugrul@etm.pdx.edu

Dundar F. Kocaoglu

Portland State University

Follow this and additional works at: https://pdxscholar.library.pdx.edu/etm_fac

Part of the Operations Research, Systems Engineering and Industrial Engineering Commons Let us know how access to this document benefits you.

\section{Citation Details}

Alanazi, H.A.; Daim, T.U.; Kocaoglu, D.F., "Identify the best alternatives to help the diffusion of teleconsultation by using the Hierarchical Decision Model (HDM)," in Management of Engineering and Technology (PICMET), 2015 Portland International Conference on , vol., no., pp.422-432, 2-6 Aug. 2015

This Article is brought to you for free and open access. It has been accepted for inclusion in Engineering and Technology Management Faculty Publications and Presentations by an authorized administrator of PDXScholar. Please contact us if we can make this document more accessible: pdxscholar@pdx.edu. 


\title{
Identify the Best Alternatives to Help the Diffusion of Teleconsultation by Using the Hierarchical Decision Model (HDM)
}

\author{
Hamad A. Alanazi, Tugrul U. Daim, Dundar F. Kocaoglu \\ Portland State University, Engineering and Technology Management Dept. Portland, OR - USA
}

\begin{abstract}
Healthcare is a very important issue in the U.S. and in the entire world and the total healthcare spending in the United States has increased over the years and by the end of 2021 it will reach up to $20 \%$ of the U.S. GDP. The increase of health expenditure, a growing U.S. population, and a projected physician shortage are the top three trends in healthcare industry. The literature highlights that researchers from different fields and with several interests recognize the important role of telemedicine in the healthcare industry. Teleconsultation is an advanced telemedicine application that offers great promise to address some of the challenges raised by these trends, and it is increasingly being included in discussions of medicine's future. Telemedicine faces many challenges in legislation and regulation, finance, technical, ethical and culture, but still the future is squarely on telemedicine. Technology Acceptance Model (TAM) is integrated with Hierarchical Decision Model (HDM) to identify the best alternatives to help the diffusion of teleconsultation.
\end{abstract}

\section{INTRODUCTION}

Healthcare is a very important issue in the U.S. and in the entire world. The U.S. spends about $18 \%$ of its GDP on healthcare, which is more than what any other developed country currently spends. Also, the USA has led the world in both the level and growth rate of spending on healthcare. The US government suffers from rising healthcare costs and in many cases these increases are passed on to American families by paying higher premiums or out-of-pocket spending. Moreover, a growing population and a shortage of physicians are complicating the healthcare issue with more challenges. The objective of this paper is to introduce one application of telemedicine, which is teleconsultation as a tool to address some of these challenges. Hierarchical Decision Model (HDM) is proposed in order to measure the teleconsultation adoption barriers that limited its advantages and to identify the best alternatives to help the diffusion of teleconsultation.

\section{A. The increase of health expenditure}

According to statistics compiled by researchers at the Centers for Medicare and Medicaid Services (CMS) the total U.S. healthcare spending reached $\$ 2.8$ trillion in 2012 , or $\$ 8,915$ per person [1]. The health spending grew $3.7 \%$ in 2012 , consistent with the same rate of growth in 2009, 2010 and 2011 [1]. National health spending remains nominal, Gross Domestic Product (GDP) grew similarly in 2010 and 2011, and health spending as a share of GDP has remained stable from 2009 through 2011, at $17.9 \%$ as shown in Fig. 1 (blue curve).
The total healthcare spending in the United States has increased over the years from $\$ 75$ billion in 1970 to $\$ 2.6$ trillion in 2010 as shown in Fig. 1 (red curve). Nevertheless, healthcare expenditure will keep increasing and according to recent study by the Center for Medicare and Medicaid Services (CMS) it will reach $\$ 4.8$ billion by the end of 2021 and that is about one-fifth or $20 \%$ of the U.S. GDP [1].

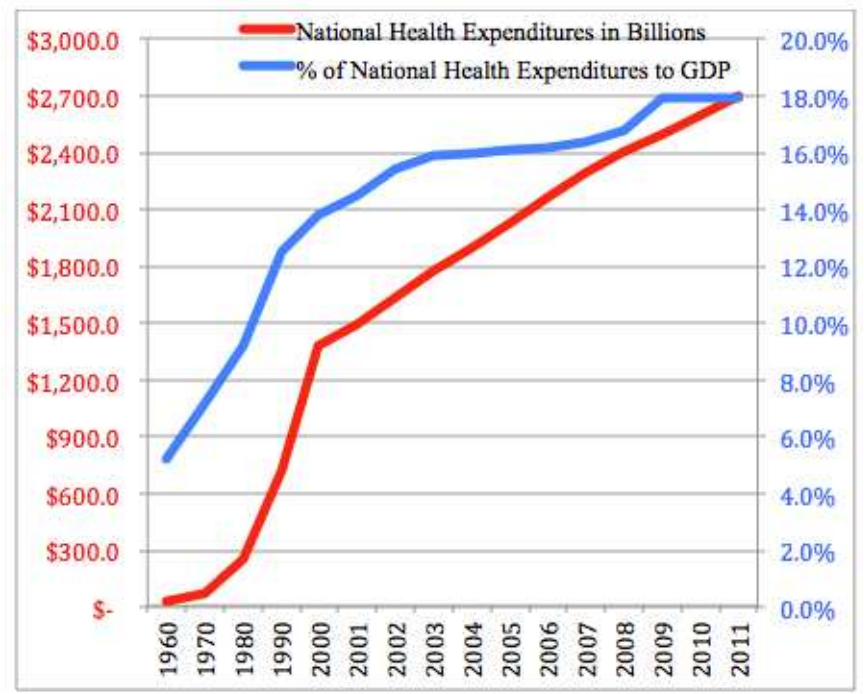

Fig. 1: national health expenditures in billions (red) and \% of national health expenditures to GDP (blue) between 1960 and 2011.

\section{B. A growing U.S. population}

According to the U.S. Census Bureau, between 2013 and 2023 the country's population will increase by 9.5 percent [2] Also, in the same study the Census Bureau projects that the population aged 65 and older will increase by nearly 45 percent [2]. As the elder population increases, the need for healthcare services and products associated with the increased prevalence of complex medical conditions and chronic diseases will continue to rise.

According to another study, by 2050 the total elder population (65 years and over) will reach above 88 million or more than double the 40.5 million elderly people in 2010 [3]. Also, by 2050 the total elder population (65 years and over) will reach above $20 \%$ ( 88 million) of the total population of the country, projected to reach more than 439 million as shown in table 1 [2]. Moreover, by 2023 about an additional 28 million people will have health insurance, according to an estimate done by the Congressional Budget Office. 
2015 Proceedings of PICMET '15: Management of the Technology Age

TABLE 1: PROJECTIONS AND DISTRIBUTION OF THE TOTAL POPULATION BY AGE BETWEEN 2010 AND 2050. SOURCE: U.S. CENSUS BUREAU, 2010.

(Numbers in thousands)

\begin{tabular}{|c|c|c|c|c|c|}
\hline Age & 2010 & 2020 & 2030 & 2040 & 2050 \\
\hline \multicolumn{6}{|l|}{ Muanen } \\
\hline Total $\ldots \ldots \ldots \ldots \ldots \ldots \ldots \ldots$ & 310,233 & 341,387 & 373,504 & 405,655 & 439,010 \\
\hline Under 20 years . .................... & 84,150 & 90,703 & 97,682 & 104,616 & 112,940 \\
\hline 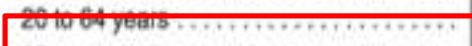 & 105,0004 & 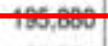 & 200,729 & 240,000 & 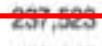 \\
\hline 65 years and over ...................... & 40,229 & 54,804 & 72,092 & B1,238 & 88,547 \\
\hline 65 to 69 years ........................ & 12,261 & 17,861 & 20,381 & 18,989 & 21,543 \\
\hline 70 to 74 years ........................ & 9,202 & 14,452 & 18,404 & 17,906 & 18,570 \\
\hline 751079 years ........................ & 7,282 & 9,656 & 14,390 & 16,771 & 15,964 \\
\hline 80 to 84 years . ...................... & 5,733 & 6.239 & 10,173 & 13,375 & 13,429 \\
\hline 85 to 89 years ....................... & 3,650 & 3,817 & 5,383 & 8,450 & 10,303 \\
\hline 90 years and over ..................... & 2,101 & 2,780 & 3,362 & 5,748 & 8,738 \\
\hline \multicolumn{6}{|l|}{ PERCENT } \\
\hline 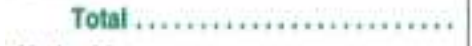 & 100.0 & 100.0 & 100.0 & 100.0 & 100.0 \\
\hline Under 20 years ...................... & 27.1 & 26.6 & 26.2 & 25.8 & 25.7 \\
\hline 20 in 64 years & 59.9 & 57.4 & 54.5 & 542 & 54.1 \\
\hline 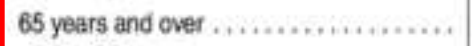 & 13.0 & 16.1 & 19.3 & 20.0 & 20.2 \\
\hline 651069 years...................... & 4,0 & $5: 2$ & 5.5 & 4.7 & 4.9 \\
\hline 70 to 74 years....................... & 3.0 & 42 & 4.9 & 4.4 & 4.2 \\
\hline 751079 years......................... & 2.3 & 2.8 & 3.9 & 4.1 & 3.6 \\
\hline 80 to 84 years ......................... & 1.8 & 1.8 & 2.7 & 3.3 & 3.1 \\
\hline 85 to 89 years. ......................... & 1,2 & 1.1 & 1.4 & 2.1 & 2.3 \\
\hline 90 years and over.................. & 0.7 & 0.8 & 0.9 & 1.4 & 2.0 \\
\hline
\end{tabular}

\section{A projected physician shortage}

The United States is likely to face a growing shortage of physicians and according to new study the U.S. will need more doctors by 2025 driven by an aging population and increased access to health insurance. Overall, the researchers projected the demand for primary care services or family doctors will grow by approximately 14 percent between 2013 and 2025 [3].

The American Association of Medical College Center (AAMC) for Workforce Studies reports the demand for physician services is increasing rapidly as the nation's population continues to age, grow, live longer and have more chronic illnesses that require increased medical attention. Also, about one in three physicians in the United States is 55 years or older and nearing retirement age [4].

TABLE 2: PHYSICIAN SUPPLY AND DEMAND PROJECTIONS, 2008 -

\begin{tabular}{|c|c|c|c|}
\cline { 2 - 4 } \multicolumn{1}{c}{} & \multicolumn{2}{c}{ Supply } & \multicolumn{2}{c|}{ Demand } & Shortage \\
\hline $\mathbf{2 0 0 8}$ & 699,100 & 706,500 & 7,400 \\
\hline $\mathbf{2 0 1 0}$ & 709,700 & 723,400 & 13,700 \\
\hline $\mathbf{2 0 1 5}$ & 735,600 & 798,500 & 62,900 \\
\hline $\mathbf{2 0 2 0}$ & 759,800 & 851,300 & 91,500 \\
\hline $\mathbf{2 0 2 5}$ & 785,400 & 916,000 & 130,600 \\
\hline
\end{tabular}

Moreover, the Association of American Medical Colleges (AAMC) projected the shortage of physicians based on supply and demand patterns, will reached over 130,000 by 2025 as shown in table 2 [5] and Fig. 2.

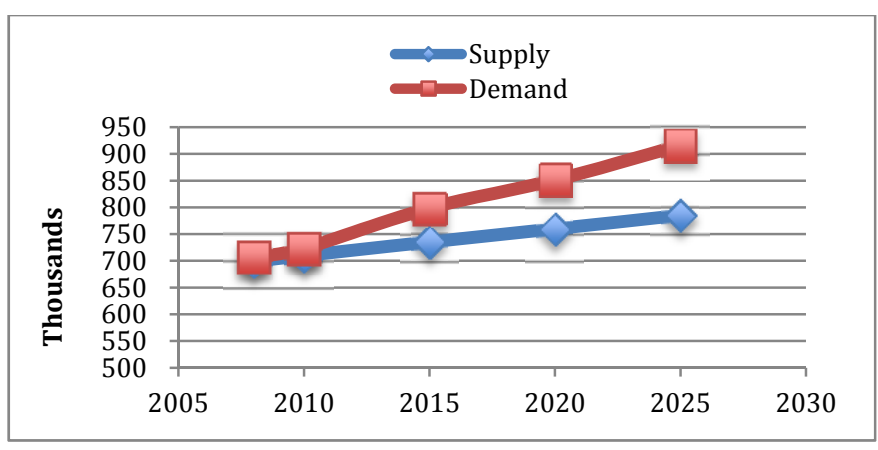

Fig. 2: physician supply and demand projections, $2008-2025$

\section{TELEMEDICINE}

Telemedicine offers great promise to address some of the challenges raised by these trends, and it is increasingly being included in discussions of medicine's future. Telemedicine offers the capabilities to deliver healthcare across distances at reduced costs while maintaining or even increasing the quality of treatment and services. Telemedicine refers generally to the use of advanced information and communication technologies (ICTs) in delivering and 


\section{Proceedings of PICMET '15: Management of the Technology Age}

supporting healthcare services. Over the past few years, it has been defined and described in several ways.

However, the term telemedicine has a much broader meaning for many researchers. It encompasses the whole range of medical applications and services including prevention of disease, diagnosis and treatment, continuing education of healthcare providers and consumers, and allows patient access to expert advice and information no matter where the patient or relevant information is located [6].

The American Telemedicine Association defined telemedicine as "the use of medical information exchanged from one site to another via electronic communications to improve patients' health status" [7].

\section{TYPES OF TELEMEDICINE}

Telemedicine can be divided according to modes of operation and according to applications.

\section{A. Modes of Operation}

Telemedicine divided according to modes of operation into three main categories [8]:

a) Store and forward (asynchronous communication): Medical data such as images or videos can be saved and then transmitted to a physician or medical specialist for assessment. Unlike traditional in-person patient meetings and actual physical examination, this method of telemedicine does not require the presence of doctor and patient at the same time. Pathology, dermatology and radiology are common specialties that use this process.

b) Real time (synchronous communication):

This division of telemedicine services involves real-time interactions between patient and healthcare provider through two-way television or phone conversations. Many traditional physical examination activities that are performed in face-to-face visits can be performed in this way. This approach requires advanced equipment and medical devices to send and receive real-time medical data and employs video conferencing.

c) Remote monitoring (self-monitoring/testing)

This method enables the clinician to monitor and measure patient health data and information remotely by using various devices. It has been used together with advanced communication equipment, for managing chronic diseases such as congestive heart failure (CHF), chronic obstructive pulmonary disease (COPD), diabetes, and asthma or to achieve earlier and proactive interventions in disease. Sensors such as blood pressure (BP), weight, pulse oximetry, glucose and ECG can be utilized for remote monitoring of patients.

\section{B. Applications}

Moreover, telemedicine applications have been characterized into four separate types [9]:

\section{a) Teleconsultation}

This uses telecommunication and information technology (ICT) to obtain the opinion of a healthcare provider who is distant from the patient and/or their primary healthcare provider, regarding the diagnosis and treatment of the patient. This type of patient/ doctor communication can take place in real time (live consultation) via a videoconferencing link. Data consultation or store-andforward process is the other form of teleconsultation, which is effected by acquiring the patient's medical information and then sending it to the consultant to give his/her opinion at some time later.

b) Telemonitoring (biotelemetry)

This is defined as the use of audio, video and other telecommunication and information technology to monitor the status of a patient at a distance [10]. It has been used to transmit the physiological and biological data of patients from one location, such as the home, office and primary clinic to another for data analysis and decisionmaking. Recently, this method has spread across the world as it has proved to be an effective tool in follow-up treatment and chronic disease management, especially in communities that have rising elderly populations. It has also been adopted as responses to the rising needs for home care [10].

c) Telesurgery (remote surgery)

It involves the manipulation of the surgical instruments by a surgeon with the assistance of advanced medical devices and telecommunication technology at a remote site from the patient [11]. A robotic system controlled by a remote surgeon directly implements all surgical tasks in real time through an interface and is as precise as traditional surgery.

\section{d) Teleeducation (telelearning)}

It has been used for many years to deliver continuing education programs to rural healthcare professionals or those with poor access such as in developing countries [12]. It has three basic modes that are video, audio and computer. Distance learning can be used in either synchronous or asynchronous modes. Video conferencing and interactive television are examples of synchronous video learning and offer live visual and verbal interaction between instructors and learners. The transmission of the spoken word (voice) can be considered as synchronous audio learning [12]. Recently, computer aided learning has become common in distance learning manifesting through many forms such as the Internet, and email.

\section{TELECONSULTATION}

Teleconsultation is an advanced telemedicine application that has spread considerably, especially in developed countries, in recent years. Teleconsultation has been defined in many ways. Essentially, consultation means seeking advice or information from somebody. Thus, teleconsultation means seeking medical advice or information from a person at a 


\section{Proceedings of PICMET '15: Management of the Technology Age}

distance [13]. This process may take place between healthcare professionals or between patient and physician. Teleconsultation is also defined according to the applied mechanism between health service providers and consumers as 'the consultation of one (or more) distant healthcare professional(s) by a locally present healthcare professional about a patient's case, diagnosis and treatment using telecommunication and information technology to bridge the spatial distance between two (or more) participants' [14]. Others have defined the teleconsultation concept in terms of its main purpose of providing medical expertise or treatment to places where there are shortages in expertise by leveraging electronic communications [15]. The clinical aims of teleconsultation are as follows [16]:

- To help make a diagnosis on the basis of clinical history, clinical examination, and investigation.

- To ask the best way to treat a patient and what that treatment should consist of.

- To obtain advice about technical aspects of care or treatment.

- To ask for corroborative opinion in relation to a recommended action for a patient.

- To obtain advice if a patient needs to transfer elsewhere for treatment and how this transfer can be done safely.

\section{A. Types of Teleconsultation}

Teleconsultation can be distinguished into two main categories: Synchronous (real-time consultation) and Asynchronous (store and forward consultation).

a) Synchronous (real-time consultation):

It involves real-time face-to-face contact (image and voice) via video-conferencing equipment such as television, digital camera, webcam or videophone to connect medical experts and clients, who are separated geographically, simultaneously [17]. It simulates the traditional physical face-to-face consultation and parties can interact about their concerns without any delay in time or data transmission. There is evidence that on-lineconsultation has proved effective in treating chronic and severe disease. Compared with asynchronous teleconsultation, this method is expensive, as it requires more advanced equipment and medical devices to send and receive real-time medical images and data and to make video link.

b) Asynchronous (store and forward consultation).

In this approach all the clinical information of the patient (medical record, video and audio recordings) and medical images are captured, processed and analyzed and sent to consultant(s) in other locations for a medical opinion. This process does not require the patient and consultant to be present at the same time or face-to-face. Subsequently, the decision is transmitted by the consultant. Recently asynchronous consultation has been divided into message, storage and discussion systems. The message system utilizes email to enable doctors to send questions directly to other specialists and receive replies. Storage systems are including Web or picture archive communication systems (PACS), which are used in teleradiology. Discussion systems implement the functionality of a typical Web-based bulletin-board system (including messaging, discussions, image storage, etc.) and typically are open to all participants [18].

Telephone is the early form of teleconsultation and is still popular in medical care. It is the cheapest of the teleconsultation methods and can be utilized within communities that are less advanced in telecommunication and information technology or are not able to afford the cost of real-time interaction.

Teleconsultation has proven its value in healthcare delivery systems and is being applied across many medical practices. Healthcare professionals and patients both benefit from teleconsultation because it is more efficient with their time. Substituting conventional faceto-face contact with video consultation becomes irresistible, particularly over large geographies, with hard to access communities or care groups, where clinical expertise is scarce and distributed. The circumstances where access to high-speed broadband is becoming available in domestic and communal environments should make this technology widespread over the next few years.

\section{B. Advantages of Teleconsultation}

Teleconsultation is, at its core, a way to deliver two important changes to healthcare [19]:

\section{a) Removes distance instantly:}

Healthcare professionals and patients are brought together through teleconsultation without having to be in the same location, thus avoiding unnecessary travel and reduced cost. It allows delivering greater levels of productivity and flexibility to healthcare providers. A patient and nurse can attend a hospital outpatient consultation "virtually" from a GP surgery through this technology. Teleconsultation can provide great benefit especially where expertise is limited and an instantaneous informed input from that expert can materially affect the treatment plan and thus the outcome for the patient.

b) Increased choices as to how services are accessed:

Healthcare providers and patients will have a wider menu of effectiveness and convenience options to work with. Teleconsultation can be best mode for change from one physical channel for accessing services to a choice of channels of coordination between healthcare professionals, their patients and care teams.

c) Other advantages of teleconsultation can be summarized as follows

- Improved quality of diagnosis and treatment through utilizing medical expertise and hence improving the quality of healthcare [14].

- Reduced diagnostic errors.

- Equality of access to specialists. 
- Improved follow-up treatment to manage chronic disease.

- Enhanced patient self-care.

- Improved patient confidence in diagnosis and treatment.

- Reduced cost for patient by avoiding unnecessary travel and for health system by eliminating waste of resources.

- Increased access to care and reduced burden on hospitals.

- Knowledge transfer.

- Increased medical education.

\section{Teleconsultation for older (geriatric) patients}

The increasing number of the elderly leads to an increase in chronic disease and multi-morbidity and for that we are focusing in this proposal only in older patients. Monitoring will become a central part of the treatment concept for many of the patients. Especially in many rural and remote regions, the spatial distribution of healthcare providers is already sparse and with a decreasing population the provision of medical care, close to the residence of the patients will be difficult. Increasing distances will restrict regular visits to practices and clinics for older patients with limited mobility. Telemedicine has the potential to improve their access to healthcare. Also, telemedicine has been used successfully with all age groups across the lifespan. Teleconsultation programs can serve an important function in home healthcare to support older adults in their own homes and communities.

\section{LITERATURE REVIEW}

The literature highlights that researchers from different fields and with several interests recognize the important role of telemedicine in the healthcare industry. There are many researchers conducting studies in order to recognize the barriers that affect the adoption and use of telemedicine systems. In this proposal we focus only in one type of telemedicine application, which is the teleconsultation. There is several barriers impact the adoption of telemedicine application through perceived usefulness and perceived ease of use, which are the two important factors of the Technology Acceptance Model (TAM).

\section{A. Technology Acceptance Model (TAM)}

User acceptance of technology has been an important field of study for over two decades. Emerging information technology cannot deliver improved organizational effectiveness if it is not accepted and used by potential users. Fred Davis and Richard Bagozzi proposed the Technology Acceptance Model (TAM) by developing the Theory of Reasoned Action (TRA), which was formulated by Fishbein and Ajzen [20]. According to the model, the acceptance of a new technology by a user is based on two factors: Perceived Usefulness (refers to how much the user believes that the technology will help to improve the performance/efficiency) and Perceived Ease of Use (refers to what extent the user is comfortable is using the features of the technology) as shown in Fig. 3 below:

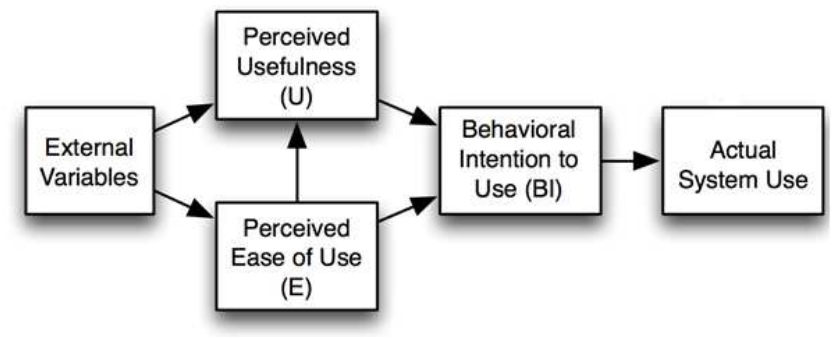

Fig. 3: final version of Technology Acceptance Model (TAM)

Teleconsultation holds great promise for new, costeffective, high quality healthcare services, and efficient methods of delivering healthcare across geographic distances. However, these benefits are constrained by many barriers and we can group them into five main barriers: financial barriers, technical barriers, logistical barriers, ethical barriers and cultural barriers.

\section{B. Financial Barriers}

Using limited financial and human resources to provide high-quality care efficiently for an increasing number of patients is a major challenge to many healthcare systems. Implementing a telemedicine program is constrained by many financial barriers such as the start-up and ongoing cost. The financial barriers include everything related to the financial issue involved in implementation of telemedicine.

\section{a) Start-up Cost}

Include initial fixed cost of the hardware, software, and technical assistance necessary to install the system, licensing fees, and the expense of maintaining the system [21].

b) Ongoing Cost

Include all the financial payments after implementing telemedicine in order to work effectively and efficiently [22].

\section{Technical Barriers}

Adopting of any telemedicine system has many technical barriers such as computer skills, training and technical support, and infrastructure. The technical barriers include everything that is related to the technical issue involved in implementation of telemedicine.

\section{a) Computer Skills}

Sufficient skills in technical and computer that are required for applying telemedicine [23].

\section{b) Training}

Includes the training needed for using the system effectively and efficiently [24].

c) Technical Support 
Includes all the technical support needed after implementing the system[25].

d) Infrastructure

Includes the network to connect to the Internet, highspeed, secure, private, and reliable [25].

\section{Logistical Barriers}

There are several logistical barriers limiting the widespread use of telemedicine systems such as licensure, credentialing, malpractice, and reimbursement. The logistical barriers include all the regulation issues involved in implementation of telemedicine.

\section{a) Licensure}

Includes all the legal documents to practice the art and science of medicine [26].

\section{b) Credentialing}

Includes all rules that allow hospitals to use telemedicine services for their patients [27].

\section{c) Malpractice}

Includes all the medical professional liability of practicing telemedicine [28].

\section{d) Reimbursement}

Include all the financial incentives for using the system [29]. Telemedicine is constrained by healthcare reimbursement processes, as both public and private insurance may not pay for telemedicine services or may pay only for some services under limited circumstances.

\section{E. Ethical Barriers}

Adopting of any telemedicine system will bring this question: whether the practice of telemedicine increases or decreases the level of patient care from an ethical point of view. The main ethical issues focus on the impact of telemedicine on the patient physician relationship and whether telemedicine increases the quantity and quality of patient care. The ethical issue that rising in adoption of telemedicine such as privacy and security, beneficence and justice.

\section{a) Privacy \& Security}

Includes the privacy and security of all personal medical data [30]. Privacy and security for personal health information mandated at the federal level by The 1996 Health Insurance Portability and Accountability Act (HIPAA).

\section{b) Beneficence}

Beneficence involves the obligation to prevent and remove harms and to promote the good of the person by minimizing possible harms and maximizing possible benefits [31].

\section{c) Justice}

Justice recognizes that each person should be treated fairly, equitably, and be given his or her due. Justice dictates that patients should be treated in a similar manner if at all possible [31].

\section{F. Cultural Barriers}

Culture barriers limiting the use of telemedicine application include the physician acceptance and patient satisfaction.

\section{a) Physician Acceptance}

The teleconsultation model depends largely on acceptance by healthcare providers (physicians, nurse practitioners, and physician assistants) [32]. We should recognize that user acceptance is important, but physician acceptance is critical [33]. Physicians are the most important users of telemedicine technology and their acceptance and satisfaction will play a crucial role in further acceptance of telemedicine [34]. Physician acceptance includes everything related to their discomfort of using the new technology equipment in their daily practices and treating patients at a distance.

\section{b) Patient Acceptance}

Telemedicine can have a positive impact on patient behavior towards managing their illness when it is feasible, reliable and the user is satisfied with the system. Patient acceptance of teleconsultation service includes anything that could decrease their acceptance of using the system.

c) Patient Satisfaction

"Satisfaction is an accepted indicator of the performance of a health- care service. It reflects patients' values and expectations regarding various aspects of a health service" [35]. Patients are satisfied when there is a match between the care expected with what is received. Patient satisfaction includes anything that could decrease their satisfaction with using telemedicine.

\section{G. Alternative Solutions}

There are several alternative solutions to address these adoption barriers. Some of the alternative solutions related to the regulations and standardized processes to implement the teleconsultation system. Government and individuals play the main role to success any adoption. After reviewing the literature we identified eight possible solutions as following:

\section{a) Government Support}

Includes all governmental support and help to adopt the system. The government support can take place in several ways such as providing some grant for telemedicine projects, providing technical assistance and developing some policies to encourage the use of telemedicine applications. Also, by evaluating the use of existing telemedicine technologies and programs and providing some recommendations to enhance the adoption.

b) Continues Training

Includes all types of training and help to use the system effective and efficient. All technology requires training and installing videoconferencing equipment in a hospital or health center need sufficient training to encourage or maintain the use for telemedicine. Telemedicine staff 
must be properly trained not just in how to turn on the equipment, but also in how to use it effectively for consultation, education and administrative purposes. Training needs to be continual, practical and in different levels with clear straightforward protocols and userfriendly instruction manuals.

c) Develop Guidelines for the Use of Telemedicine

Includes all guidelines and regulations to use the system. As the number of physicians using telemedicine to diagnose and treat patients is growing, it is essential to develop guidelines to provide states a framework to use when creating their own telemedicine laws. The guidelines should cover the evaluation and treatment of the patient, informed consent, continuity of care, referrals for emergency services, medical records, privacy and security of patient records and exchange information, disclosures and functionality on online services making available telemedicine technologies, and prescribing.

d) Multi-State License for Telemedicine

Includes all licenses for physicians to practice across state borders. Physician practice is regulated in each state by a state medical board whose authority is granted by the state legislature. Each state board functions independently of other states and this led to variations in policies from state to state that physicians will need to understand and obey if they practice in multiple states. Multi-state telemedicine license will address all of the variations policies and make it easier for physicians to practice across state borders in multiple states. That would allow a physician to apply for the multi-state license through his or her state of principal licensure.

e) Multi-State Standards and Regulation

Includes all standards and regulations to adopt the system. The multi state standards and regulations provide uniform quality of service to patients, and to promote reasonable and informed patient and provider expectations. The purpose of these standards and regulations is to assist physicians to provide effective and safe medical care for patient needs. These standards and regulations are fundamental requirements to be followed when providing medical and other healthcare services using telecommunications technologies, and any other electronic communications between patients, practitioners and other healthcare providers.

\section{f) Standard Processes for Reimbursement}

Includes all reimbursement standards and regulations for using the system. Reimbursement is very important in order to encourages use of telemedicine services, provides mechanism to reimburse providers and as a tool to ensure sustainability of program. Federal and state policies on reimbursement could lead to more helpful policies for government and private payers. Reimbursement standards help on the billing such as the billing codes for telemedicine services and how telemedicine services reimbursement.

\section{g) Ethical Codes}

Includes all medical and other professional ethical codes to use and adopt the system. There should be ethical and professional codes to all aspects of a telemedicine to follow. All physicians must respect the ethical guidelines and codes when practicing telemedicine such as the confidential relationship between patient-physician and quality of care.

h) Education

Includes all types of education that help to adopt the telemedicine system. Education is very important in healthcare service and can take place in several ways such educate patients to use the telemedicine programs and their advantages by using such telemedicine program. Also, it could take place as a short courses or seminar, workshop and face-to-face or online.

\section{RESEARCH FRAMEWORK}

\section{A. Research Objectives (RO)}

The proposed research will address the research objective below:

RO: Develop a Hierarchical Decision Model (HDM) to identify the best alternatives to help the diffusion of teleconsultation.

\section{B. Research Questions (RQ)}

The importance of telemedicine and its applications in the healthcare industry is well recognized. From all that has been previously discussed, it becomes imperative to increase empirical research in order to better understand the barriers that affect the adoption and use of teleconsultation applications. The following sixteen research questions have been identified in order to address these gaps.

RQ1: How do perceived usefulness and perceived ease of use impact the diffusion of teleconsultation?

RQ2: Which barriers have more effect on the adoption through perceived usefulness?

RQ3: Which barriers have more effect on the adoption through perceived ease of use?

RQ4: Which financial barriers have more effect on the diffusion of teleconsultation?

RQ5: Which technical barriers have more effect on the diffusion of teleconsultation?

RQ6: Which logistical barriers have more effect on the diffusion of teleconsultation?

RQ7: Which ethical barriers have more effect on the diffusion of teleconsultation?

RQ8: Which culture barriers have more effect on the diffusion of teleconsultation?

RQ9: How does government support help the diffusion of teleconsultation?

RQ10: How does training help the diffusion of teleconsultation?

RQ11: How does multi-state license help the diffusion of teleconsultation? 


\section{Literature Review to Research Goal and Questions}

\begin{tabular}{|l|l|}
\hline \multicolumn{1}{|c|}{ Literature Review } \\
\hline $\begin{array}{l}\text { The diffusion of teleconsultation } \\
\text { impacted by perceiven usefulness and } \\
\text { perceived ease of use. }\end{array}$ \\
\begin{tabular}{|l|l|} 
Implementing teleconsultation \\
constrained by many financial barriers \\
such the start-up and ongoing cost.
\end{tabular} \\
\hline $\begin{array}{l}\text { Adopting of teleconsultation has many } \\
\text { technical barriers such computer skills, } \\
\text { training and technical support, and } \\
\text { infrastructure }\end{array}$ \\
\hline $\begin{array}{l}\text { There are several logistical barriers } \\
\text { limited the widespread of } \\
\text { teleconsultation such as licensure, } \\
\text { credentialing, malpractice, and } \\
\text { reimbursement. }\end{array}$ \\
\begin{tabular}{|l|l|l|}
\hline $\begin{array}{l}\text { Ethical barriers limited the use of } \\
\text { teleconsultation, which include privacy } \\
\text { \& security, beneficence and justice. }\end{array}$ \\
\hline $\begin{array}{l}\text { Culture barriers limited the use of } \\
\text { teleconsultation, which include the } \\
\text { physician and patient acceptance and } \\
\text { patient satisfaction. }\end{array}$ \\
alternatives to help the diffusion of \\
teleconsultation.
\end{tabular} \\
Mow do perceived usefulness and \\
perceived ease of use impact the
\end{tabular}

RQ12: How do telemedicine guidelines help the diffusion of teleconsultation?

RQ13: How do multi-state standards and regulations help the diffusion of teleconsultation?

RQ14: How do reimbursement standard processes help the diffusion of teleconsultation?

RQ15: How does ethical code help the diffusion of teleconsultation?

RQ16: How does education help the diffusion of teleconsultation?

Six literature review gaps have been identified, sixteen research questions have been developed, and one-research objective established. The literature review, research questions, and research objectives are summarized in table 3.

\section{Research Model}

After reviewing the literature in telemedicine adoption we grouped the adoption barriers to five main barriers: financial barriers, technical barriers, logistical barriers, ethical barriers and cultural barriers. Each one of these five adoption barriers has sub-barriers. The Hierarchical Decision Model (HDM) looks like a good technique to solve this multi-criteria prioritization problem. Also, the Technology Acceptance Model (TAM) explains how a new technology and the various aspects of it are received and used by the user. The proposed model is an integration of The Hierarchical Decision Model (HDM) and Technology Acceptance Model (TAM).

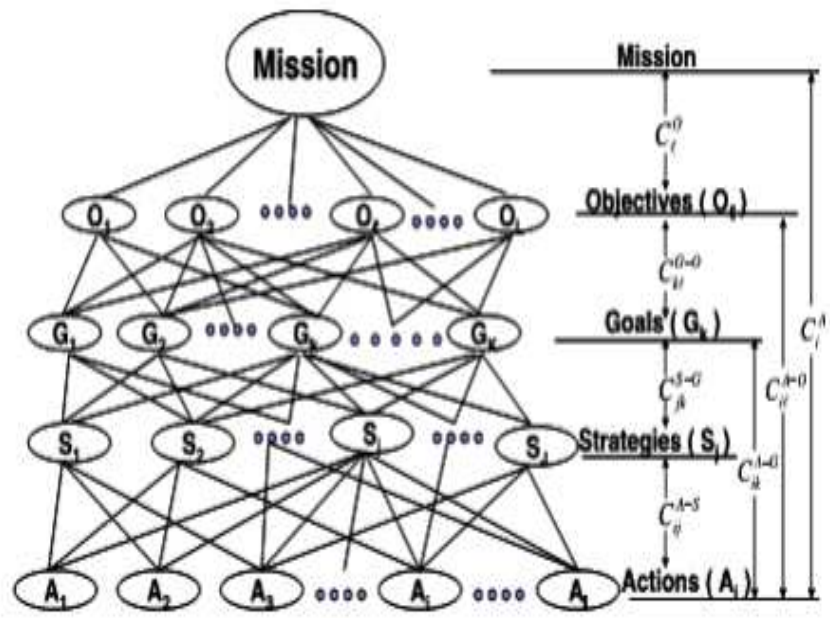

Fig. 4: five levels of the Hierarchical Decision Model (HDM)

\section{Hierarchical Decision Model (HDM)}

The Hierarchical Decision Model (HDM) structure was first used by Dr. Kocaoglu to solve complex multi-criteria decision problems $[36,37]$. For any hierarchical decision model, the basic structure of the hierarchy is presented in the 
MOGSA form. This model consists of five levels of decision elements namely Mission, Objectives, Goals, Strategies and Actions as shown in Fig. 4 [38, 39]. The process requires the decision maker to provide judgments about the relative importance of each of the criteria and then to specify a preference for each decision alternative on each criterion [40].

\section{E. Integrated Model}

The output of the HDM model is a prioritized ranking indicating the overall preference for each of the decision alternatives. Also, HDM can be used to solve prioritization problems. To address the research objectives and research gaps, a theoretical research model is developed as shown in Fig. 5. The proposed research model is based on a literature review of barriers related to the adoption and use of the teleconsultation. Also, the proposed research model is based on a literature review of HDM and TAM models to develop an appropriate research model.

\section{RESEARCH METHODOLOGY}

A quantitative research method is proposed in order to address the research objectives. An initial research model will be developed, which contains five main barriers and sixteen sub-barriers. The research aim is to conduct an empirical research approach based on theoretical models and collect data obtained from the telemedicine experts and users.

\section{A. Data Collection}

In this research, the link to the proposed HDM model will be sent by email to the experts and people who use the telemedicine system in order to collect the data. The targeted research audience would be the experts in telemedicine system, physicians, nurses, clinical staff and patients in the Northwest. Table 4 below summarizes the data sources for the HDM model.

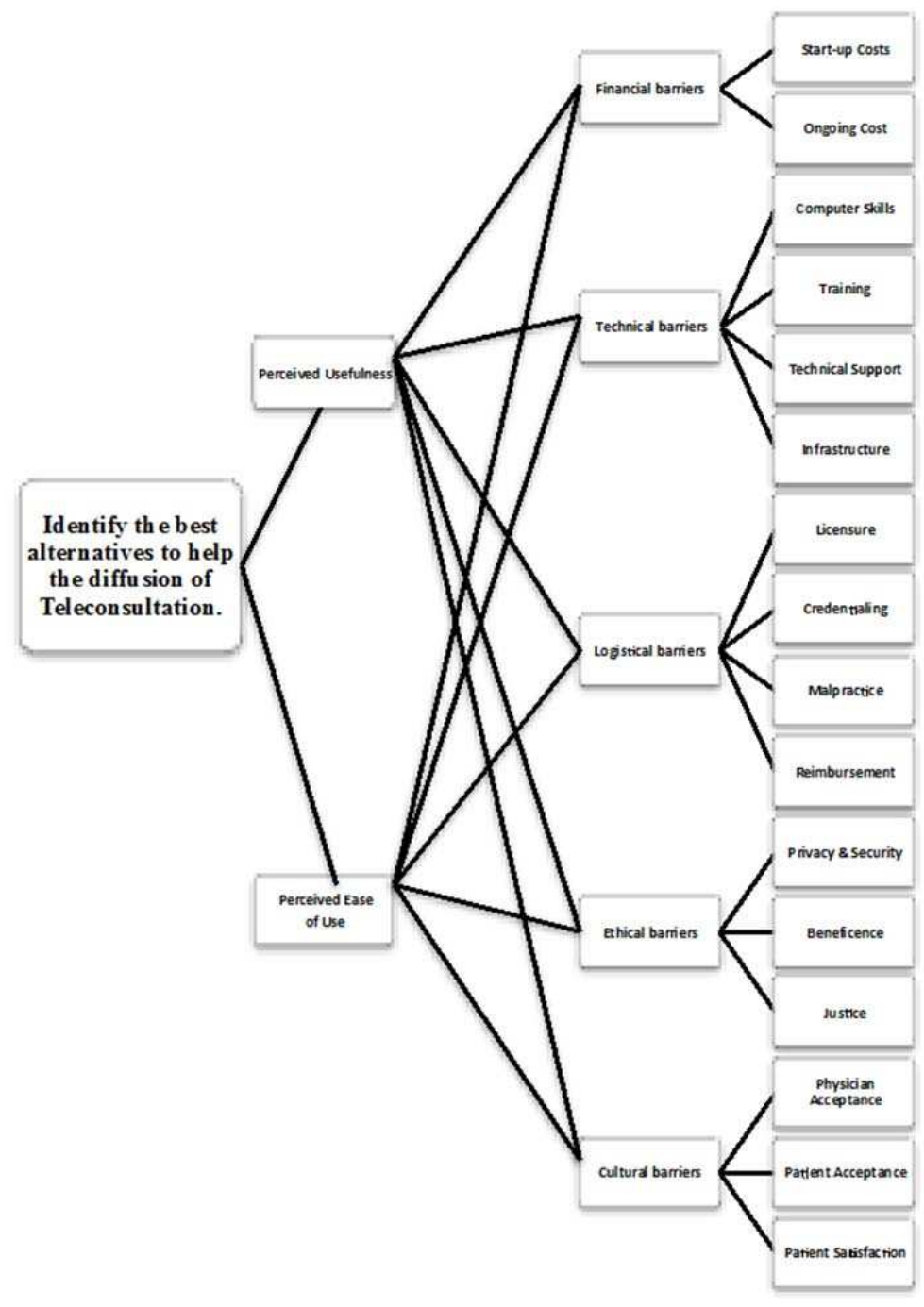

Fig. 5: proposed research model.

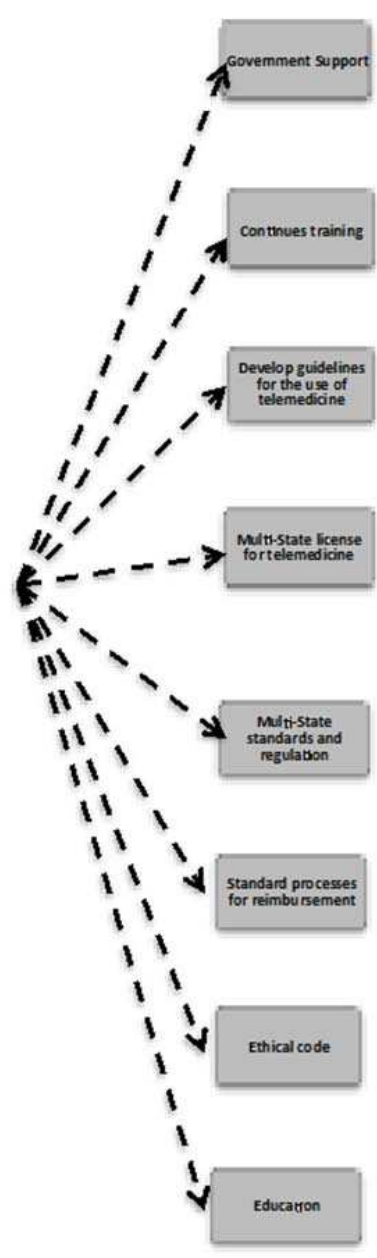


2015 Proceedings of PICMET '15: Management of the Technology Age

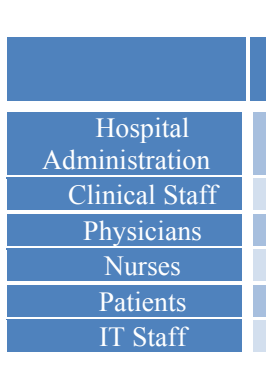

\section{B. Data Analysis}

After getting the data from experts and system users, the results will be validated and related analysis will be conducted. The experts and system users inconsistency will be tested and according to Kocaoglu's research, if the inconsistency level is less than $10 \%$ or 0.1 , the related judgmental data should be acceptable [41]. Also, the disagreement among the experts and system users will be tested and the disagreement measured based on the Ph.D. dissertation done by N. Gerdsri (2004), and then expanded by P. Gerdsri' Ph.D dissertation in 2009 [40, 42].

Moreover, the data sensitivity analysis for the hierarchical decision models will be based on Chen's dissertation and Kocaoglu's research [43, 44]. With this method, algorithms were developed based on a series of mathematical deductions. It is an accurate and comprehensive method to examine the impact of changes in different levels of a hierarchical decision model on the ranking of the alternatives. In this method, tolerance is defined as the allowable range in which a contribution value can vary without changing the ranking order of bottom level alternatives.

\section{RESEARCH CONTRIBUTIONS}

Although telemedicine has many definitions and different categories and classifications, no one can ignore its benefits for patients, physicians, and communities. Telemedicine is still a rich area for more innovations and more investments and the future will bring more opportunities as the new technologies improve the quality of telemedicine services, and at the same time realize a huge improvement of the telecommunications technology.

Telemedicine faces many challenges in legislation and regulation, finance, and also technical, ethical and culture challenges, but still the future is squarely on telemedicine. There is much evidence to support its cost benefits and improve the quality of healthcare services. Telemedicine needs support from individuals, communities, government, and healthcare providers and payers in order to be the first option to take over the traditional face-to-face practice. The proposed empirical research and the proposed HDM model is expected to provide numerous contributions to the body of knowledge:
A. Identify the best alternatives to help the diffusion of teleconsultation:

The proposed HDM model could identify the best alternative solutions to increase the diffusion of teleconsultation. By giving these alternatives more consideration we could increase the successful adoption rate.

B. Explain the reasons behind many telemedicine adoption failures:

There are many telemedicine adoption failures and by applying the proposed HDM model we will identify the top barriers that cause the failure, and give these barriers more consideration during the implementation. Also, this proposed HDM model could reduce the failure rate on adoption of telemedicine by providing early indicators for which criteria or sub-criteria needs more attention before or during the implementation or adoption of telemedicine systems.

C. Increase the successful rate of adoption.

The proposed HDM model could explain many adoption failures and we could come up with some recommendations from these failures. The proposed HDM model could increase the successful rate of adoption by identifying, from step one, which barriers we could face and which one could cause the failure. By giving these barriers more attention during or before adoption of telemedicine systems we could likely increase the probability of success of the adoption.

\section{REFERENCES}

[1] [Centers for Medicare \& Medicaid Services, Office of the Actuary, National Health Statistics Group. Retrieved 11/15/14 World Wide Web,

http://www.cms.gov/Research-Statistics-Data-and-Systems/Statistics-Trendsand-Reports/NationalHealthExpendData/downloads/tables.pdf

[2] U.S. Department of Commerce - United States Census Bureau, Retrieved 11/02/14 World Wide Web, http://www.census.gov/prod/2010pubs/p25-1138.pdf

[3] T. M. Dall, P. D. Gallo, R. Chakrabarti, T. West, A. P. Semilla, and M. V. Storm, "An aging population and growing disease burden will require alarge and specialized health care workforce by 2025," Health affairs, vol. 32, 2013.

[4] The American Association of Medical College Center (AAMC), Retrieved 10/23/14 World Wide Web https://www.aamc.org/download/100598/data/

[5] Association of American Medical Colleges, Retrieved 10/23/14 World Wide

Web https://members.aamc.org/eweb/upload/The\%20Complexities $\% 20$ of $\% 2$ 0Physician\%20Supply.pdf 
[6] R. Wootton, J. Craig, and V. Patterson, Introduction to telemedicine: Royal Society of Medicine Press, 2006.

[7] American Telemedicine Association $(2013,12)$. What is Telemedicine. Retrieved 12/17/13 World Wide Web, http://www.americantelemed.org/learn/what-istelemedicine\#.UueJmWTTky4

[8] R. Hoyt, M. Sutton, and A. Yoshihashi, Medical Informatics: Practical Guide for the Healthcare Professional 2007: Lulu. com, 2007.

[9] A. C. Norris and A. Norris, Essentials of telemedicine and telecare: Wiley Chichester, 2002.

[10] S. Meystre, "The current state of telemonitoring: a comment on the literature," Telemedicine Journal \& e-Health, vol. 11, pp. 63-69, 2005.

[11] S. Kumar and J. Marescaux, Telesurgery: Springer Science \& Business Media, 2008.

[12] V. R. Curran, "Tele-education," Journal of telemedicine and telecare, vol. 12, pp. 57-63, 2006.

[13] R. Wootton, "Realtime telemedicine," Journal of telemedicine and telecare, vol. 12, pp. 328-336, 2006.

[14] M. Nerlich and U. Schaechinger, Integration of health telematics into medical practice vol. 97: IOS Press, 2003.

[15] Khoury, N. E., "Doctors Truly Without Borders: Teleconsultation in the $21^{\text {st }}$ Century". The National Arab American Medical Association (NAAMA), Retrieved 10/27/14 World Wide Web, http://www.naama.com/pdf/doctors-without-borders-teleconsultationnabil-khoury-md.pdf

[16] A. W. Darkins and M. A. Cary, Telemedicine and telehealth: principles, policies, performances and pitfalls: Springer publishing company, 2000.

[17] F. Verhoeven, K. Tanja-Dijkstra, N. Nijland, G. Eysenbach, and L. van Gemert-Pijnen, "Asynchronous and synchronous teleconsultation for diabetes care: a systematic literature review," Journal of diabetes science and technology, vol. 4, pp. 666-684, 2010.

[18] R. Luk, M. Ho, and P. M. Aoki, "Asynchronous remote medical consultation for Ghana," in Proceedings of the SIGCHI conference on human factors in computing systems, 2008, pp. 743-752.

[19] P. Rice, "Teleconsultation for Healthcare Services. A workbook for implementing new service models," ed: Yorkshire \& Humber HIEC Bradford, UK, 2011.

[20] I. Ajzen and M. Fishbein, "Understanding attitudes and predicting social behaviour," 1980.

[21] R. L. Bashshur, T. G. Reardon, and G. W. Shannon, "Telemedicine: a new health care delivery system," Annual Review of Public Health, vol. 21, pp. 613-637, 2000.

[22] G. A. Loomis, J. S. Ries, R. M. Saywell, and N. R. Thakker, "If electronic medical records are so great, why aren't family physicians using them?," Journal of Family Practice, vol. 51, pp. 636-641, 2002.

[23] D. L. Paul, K. E. Pearlson, and R. R. McDaniel Jr, "Assessing technological barriers to telemedicine: technology-management implications," Engineering Management, IEEE Transactions on, vol. 46, pp. 279-288, 1999.

[24] C. O. Bagayoko, H. Müller, and A. Geissbuhler, "Assessment of Internet-based tele-medicine in Africa (the RAFT project)," Computerized Medical Imaging and Graphics, vol. 30, pp. 407-416, 2006.

[25] N. Barry, "Telemedicine diffusion in Scotland: training and technical support issues," British Journal of Healthcare Computing and Information Management, vol. 17, pp. 20-23, 2000.

[26] C. E. Lewis, "My Computer, My Doctor: A Constitutional Call for Federal Regulation of Cybermedicine," Am. JL \& Med., vol. 32, p. 585, 2006.
[27] R. Fitzgerald, "Medical regulation in the telemedicine era," The Lancet, vol. 372, pp. 1795-1796, 2008.

[28] C. Natoli, "Summary of findings: Malpractice and telemedicine," Retrieved Oct, vol. 5, p. 2012, 2009.

[29] B. Chaudhry, J. Wang, S. Wu, M. Maglione, W. Mojica, E. Roth, et al., "Systematic review: impact of health information technology on quality, efficiency, and costs of medical care," Annals of internal medicine, vol. 144, pp. 742-752, 2006.

[30] G. Wachter, "HIPAA's Privacy Rule Summarized: What does it mean for Telemedicine," Telemedicine Regulatory Issue Summary. Retrieved September, vol. 13, p. 2003, 2001.

[31] P. A. Clark, K. Capuzzi, and J. Harrison, "Telemedicine: Medical, legal and ethical perspectives," American Journal of Case Reports, vol. 16, pp. RA261-RA272, 2010.

[32] K. McConnochie, N. Wood, N. Herendeen, C. ten Hoopen, L. Denk, and J. Neuderfer, "Integrating telemedicine in urban pediatric primary care: provider perspectives and performance," TELEMEDICINE and eHEALTH, vol. 16, pp. 280-288, 2010.

[33] R. Latifi, Current principles and practices of telemedicine and ehealth: Ios Press, 2008.

[34] K. A. Frey and R. L. Bratton, "Role of telemedicine in the health care delivery system," The Journal of the American Board of Family Practice, vol. 15, pp. 170-171, 2002.

[35] M. Yip, A. M. Chang, J. Chan, and A. E. MacKenzie, "Development of the Telemedicine Satisfaction Questionnaire to evaluate patient satisfaction with telemedicine: a preliminary study," Journal of Telemedicine and Telecare, vol. 9, pp. 46-50, 2003.

[36] D. F. Kocaoglu, "A participative approach to program evaluation," Engineering Management, IEEE Transactions on, pp. 112-118, 1983.

[37] D. F. Kocaoglu, "Hierarchical decision modeling," Engineering Management Program, Portland State University, 1988.

[38] D. Kocaoglu and M. Guven Iyigun, "Strategic R\&D program selection and resource allocation with a decision support system application," in Engineering Management Conference, 1994.'Management in Transition: Engineering a Changing World', Proceedings of the 1994 IEEE International, 1994, pp. 225-232.

[39] T. Turan, M. Amer, P. Tibbot, M. Almasri, F. Al Fayez, and S. Graham, "Use of Hierarchal Decision Modeling (HDM) for selection of graduate school for master of science degree program in engineering," in Management of Engineering \& Technology, 2009. PICMET 2009. Portland International Conference on, 2009, pp. 535-549.

[40] P. Gerdsri and D. F. Kocaoglu, "HDM for developing national emerging technology strategy and policy supporting sustainable economy: A case study of nanotechnology for Thailand's agriculture," in Management of Engineering \& Technology, 2008. PICMET 2008. Portland International Conference on, 2008, pp. 344-350.

[41] D. F. Kocaoglu, "A systems approach to the resource allocation process in police patrol," University of Pittsburgh., 1976.

[42] N. Gerdsri and D. F. Kocaoglu, "A quantitative model for the strategic evaluation of emerging technologies," Engineering and Technology Management Department, Portland State University, Portland, OR, vol. $97201,2004$.

[43] H. Chen, Sensitivity analysis for hierarchical decision models: Portland State University, 2007.

[44] H. Chen and D. F. Kocaoglu, "A sensitivity analysis algorithm for hierarchical decision models," European Journal of Operational Research, vol. 185, pp. 266-288, 2008. 